\title{
Surface properties of hydrogenated diamond in the presence of adsorbates: a hybrid functional DFT study
}

\author{
Pablo Rivero ${ }^{1, *}$, William Shelton ${ }^{1}$, and Vincent Meunier $^{2 *}$ \\ 1. Center for Computation and Technology, Louisiana State University, Baton Rouge, Louisiana 70803, USA \\ 2. Department of Physics, Applied Physics, and Astronomy, \\ Rensselaer Polytechnic Institute, Troy, NY 12180, USA
}

(Dated: September 20, 2016)

\begin{abstract}
We use an ab initio B3LYP approximation to perform a quantitative analysis of the electronic and structural properties of hydrogen terminated (100), (110), and (111) diamond surfaces. We also study the charge transfer produced by the adsorption of a number of molecular species, including $\mathrm{NO}_{2}, \mathrm{NO}$, and $\mathrm{O}_{3}$. Our results shed light on the understanding of hydrogenated diamond surface conductivity and demonstrate that combination of surface termination and molecular species is key to control the hole doping on diamond. This study, which does not rely on any empirical parameters, also provides guidelines for the choice of adsorbate molecules that yield desired hole injection to the hydrogenated diamond surfaces.
\end{abstract}

PACS numbers:

\section{INTRODUCTION}

Diamond is one of the oldest known allotrope forms of carbon. This metastable crystal is endowed with a number of outstanding properties that can be exploited for high-power electronic applications under extreme conditions of voltage, temperature, and frequency. It has five times the operational temperature of silicon $[1,2]$ and the highest Debye temperature (2220 K), exceeding that of most other insulating materials by one order of magnitude. Due to its large electronic band gap, diamond features 20 times the breakdown field strength of mica (material commonly used in capacitors) and therefore, it can be used in high voltage insulation across thin layers. Its transport properties are characterized by a charge carrier mobility of $\simeq 3000 \mathrm{~cm}^{2} / \mathrm{Vs}[3]$ and the highest thermal conductivity of any material at room temperature $(>20 \mathrm{~W} / \mathrm{cmK})$, exceeding that of copper by a factor of five [4].

Even though these remarkable semiconducting properties have been known for decades, diamond has not yet been fully exploited in electronic and thermal applications. The lack of progress has been largely due to the limited availability of high quality substrates and its expensive and difficult processing route. Moreover, doped diamond - which is needed to assemble electronic devices - is very limited due to its extremely rigid lattice and its low chemical reactivity. The impediments related to cost and quality control have been partially overcome by recent advances in chemical vapor deposition (CVD) synthesis of nanodiamond [5]. Furthermore, hole-carrier conductivity in hydrogenated diamond (H-D) surfaces has been proven as a route to the development of diamondbased applications. In this case, charge transfer occurs for hydrogenated sites to adsorbate acceptors, leading to a highly conductive layer of holes on the diamond surface [6-9].
While experimental studies have provided evidence of the promise of H-D, many fundamental questions such as 'what kind of molecular adsorbate produces hole doping on diamond surfaces' or 'how can we control the hole density on diamond surfaces', remain to be addressed in the quest of developing the technology for using $\mathrm{H}$ $\mathrm{D}$ in consumer products. To provide insight into these questions, we performed a systematic first-principles investigation of the electronic and structural properties of different H-D surfaces. We also studied the influence of the adsorption of different candidate acceptor molecules on the electronic properties, and find that the choice of the H-D configurations and adsorbate molecules are key to controlling the hole density at the diamond surface.

Diamond surfaces show the usual positive electronic affinity when they are in a bare or oxygen terminated configuration. In contrast, H-D surfaces feature a negative electronic affinity (NEA), i.e., the vacuum level lies below the conduction band minimum (CBM). This property has been observed theoretically and experimentally [10] and is due to the formation of dipoles induced by the heteropolar $\mathrm{C}-\mathrm{H}$ bonds [11] that leads to the bending of the surface bands. In this condition, electrons at the CBM are readily transferred into the vacuum level, which promises highly attractive potential applications (e.g., efficient cathodes [12]). However, chemisorbed hydrogen is necessary but not sufficient for ensuring a high surface conductivity in diamond [13]. Indeed, the H-D surface must be exposed to air or strongly electron accepting molecules $[8,14]$ in order to produce surface conductivity. In this situation, electrons are transferred from H-D to the adsorbate resulting in a hole accumulation layer on diamond surface and therefore, a quasi two-dimensional hole gas. Note that this hole density at the surface is achieved without introducing impurities into diamond, thereby avoiding one of the shortcomings of diamond for technological applications.

Our investigation includes the study of the main 
growth forms of diamond: (100), (110), and (111) diamond surfaces. For our calculations we employed the three-parameter B3LYP $[15,16]$ hybrid functional. The use of a hybrid functional provides the first quantitative description of H-D's structural and electronic properties and for the adsorption process of different molecules, without the need of any experimental input. We consider the $\mathrm{NO}_{2}, \mathrm{NO}$, and $\mathrm{O}_{3}$ molecules, which have been previously studied and suggested as acceptor molecules for H-D surfaces [17], thus improving the surface conductivity.

In contrast to existing works which use either local or semi-local exchange correlation functionals, hybrid functionals include a portion of Hartree-Fock exchange potential that provides a better representation of the electronic properties. B3LYP has provided exceptional quantitative accuracy in the past, describing the structural and electronic properties of bulk diamond [18], bare diamond surfaces [19], and other insulating material surfaces [20-23]. This improvement is especially noteworthy with respect to the electronic properties, where local and semi-local functionals fail to provide a correct description. Note that since molecular adsorption on diamond is governed by charge transfer between the acceptor molecule and $\mathrm{H}$ $\mathrm{D}$, van der Waals corrections are not needed in the study of these systems. In this work we provide quantitatively accurate values of properties that include: structural geometry, surface energy, hydrogen desorption, electronic affinity, band bending, and band gap. Our results also provide information on the surface charge transfer produced on different H-D surfaces that can be used to guide experimental investigations targeting future development on surface hole conductivity.

\section{COMPUTATIONAL DETAILS}

The electronic and crystal structure properties of hydrogen-terminated diamond surfaces and the adsorption of different molecules have been investigated by means of a two-dimensional periodic slab model using the replicated-data version of the CRYSTAL-14 computational package $[24,25]$. CRYSTAL-14 employs atomcentered Gaussian-type orbital (GTO) basis sets to build the Bloch functions to expand the one-electron crystalline orbitals in the two- and three- dimensional crystal structures.

Two different approximations of the density functional theory (DFT) have been used in this investigation. First, we selected the Perdew-Burke-Ernzerhof (PBE) form of the generalized gradient approximation (GGA) since it is one of the most accurate examples of a semi-local functional [26]. Second, we employed the standard B3LYP hybrid functional $[15,16]$ that mixes $20 \%$ of HartreeFock with $8 \%$ of a local density approximation (VWN $[27]$ ) and $72 \%$ of a GGA functional (Becke88 [28]) in the exchange potential, while the correlation potential mixes $19 \%$ of VWN with $81 \%$ of LYP [29] GGA functional. B3LYP is ubiquitous in the study of finite size systems (e.g., molecules) due to its non-local treatment of the exchange-correlation potential. The computational cost associated with the evaluation of the exact exchange contribution is reduced from $N^{4}$ scaling to $N^{2}-N^{3}$ (where $N$ is the number of atoms making up the system) using GTOs. Thus, allowing the use of hybrid functionals in the study of the geometry and energetics of bare diamond surfaces [19] as well as other surfaces [20-23].

The all-electron GTO's considered here are the following: for $\mathrm{C}$, the 6-3111G all electron basis was chosen [30], since it has been shown to yield accurate results for bare diamond surfaces [19]. The rest of atomic basis sets used in this study were adopted from the CRYSTAL14 online repository [31] (i.e., H: 3-1p1G; N: 6-31d1G; and O: 6-2111d1G). These basis sets are known to provide accurate description of the properties of materials in similar molecular environments. For our calculations a 8 $\times 8 \times 1$ Monkhorst-Pack mesh [32] was found to yield converged energies to $<10^{-6}$ ua. Tolerances of $10^{-7}$ (ITOL1, ITOL2, ITOL3), $10^{-8}$ (ITOL4), and $10^{-18}$ (ITOL5) were used for the bielectronic Coulomb and Hartree-Fock exchange series integrals as described in Ref. 25. The total energy convergence threshold between geometry optimization steps was set to $10^{-10}$ au. Effective charges on atoms have been calculated according to the Mulliken population analysis [33], which provides an estimation of the partial atomic charges, thereby permitting semi-quantitative and comparative results.

The diamond surfaces were modeled using slabs symmetrically terminated, and therefore containing two surfaces. The total number of layers range between 8 and 16 depending on the surface termination considered. To determine the ground-state atomic positions, full atomic relaxations were performed on the first 3 to 7 surface layers depending on surface termination for bare and hydrogenated diamond systems. The innermost 2 carbon layers and cell parameters were fixed to the corresponding PBE and B3LYP calculated structural bulk properties. For the adsorption of molecular species on H-D surfaces we relaxed the outer 3,4 and 5 surface layers depending on the surface termination. This choice was based on a compromise between computational cost and properties accuracy. The hydrogen layer and the molecular adsorbate were also relaxed. In all cases, the convergence criterion on gradient components and nuclear displacements was set to $0.0003 \mathrm{Ha} / \mathrm{Bohr}$ and $0.0012 \mathrm{Bohr}$ respectively.

For bare diamond surfaces, the surface energy per area is calculated using the following expression:

$$
\gamma=\frac{E_{\text {slab }}-N \cdot E_{\text {bulk }}}{2 A}
$$

where $\mathrm{E}_{\text {slab }}$ is the total energy of the surface slab, $N$ is 
the number of atoms in the surface slab, $\mathrm{E}_{\text {bulk }}$ is the bulk energy per atom and $A$ is the surface area. For a slab, we have two identical surfaces, which accounts for the factor of two in the denominator. Hydrogen desorption energy is defined as

$$
E_{\text {desorp }}=\left(E_{D}+\frac{n_{H} \cdot \mu_{H_{2}}}{2}\right)-E_{H D}
$$

where $\mathrm{E}_{D}$ is the total energy of the bare diamond surface, $\mathrm{E}_{H D}$ is the total energy of the system containing $N$ hydrogen atoms, $\mathrm{n}_{H}$ is the number of $\mathrm{H}$ atoms on the surfaces, and $\mu_{\mathrm{H}_{2}}$ is the chemical potential of hydrogen. This potential is evaluated by calculating the energy of an isolated $\mathrm{H}_{2}$ molecule.

For these energy differences as well as for the reported cohesion energies of different adsorbates (Table VIII), the basis set superposition error (BSSE) $[34,35]$ needs to be considered. Following the procedure in Refs. 19 and 31, we used 'ghost' functions to evaluate the BSSE by means of the counterpoise method [36, 37]. Our results gave us estimated BSSE correction values in the range of -0.04 and $-0.01 \mathrm{eV}$ for all energy differences results reported in this work. We have also employed 'ghost' functions at the surface for a better description of the NEA (NEA*), as it has been reported in previous works $[38,39]$. Both calculated values will be reported.

Finally, we want to point out that CRYSTAL slab model is based on a Gaussian basis set and not planewaves, which requires periodicity in all three directions. Therefore, dipolar corrections are not required. This has the added advantage that the electrostatic potential approaches zero at large distances from the surface. It follows that the vacuum level is at $0 \mathrm{eV}$ and as a consequence, the work function is just the negative of the Fermi level. Note that unlike previous theoretical investigations that made use of the experimental bandgap of bulk diamond to quantify the NEA [40-44], our work is solely based on our calculations, thereby providing a consistent process for determining key electronic properties, interactions, and energetics. Specifically, the electron affinity has been calculated by subtracting the vacuum level from the CBM, which is taken as the Fermi energy plus the band gap.

\section{RESULTS AND DISCUSSION}

This section is organized as follows: we first address the accuracy of PBE and B3LYP functionals for describing bulk diamond properties. Next, we study the structural and electronic properties of bare and hydrogenated (100), (110), and (111) diamond surfaces. Finally, we present results for the adsorption of different molecules on the H-D surfaces and their corresponding effects on the electronic structure and charge transfer process.
Table I. Calculated PBE and B3LYP cell parameters, C-C distances $(\AA)$, and band gaps (eV) of bulk diamond. Experimental values shown for comparison have been extracted from Ref. 45.

\begin{tabular}{lccc} 
& PBE & B3LYP & EXP. \\
\hline $\mathrm{a}$ & 3.577 & 3.575 & 3.57 \\
$\mathrm{~d}_{C-C}$ & 1.549 & 1.548 & 1.546 \\
Gap & 4.00 & 5.66 & $5.46-5.60$
\end{tabular}

\subsection{Bulk diamond}

The experimental lattice constant and band gap for bulk diamond are $3.57 \AA$ and between 5.46 and $5.60 \mathrm{eV}$ [45] respectively. Table I shows that both functionals reproduce remarkably well the experimental lattice constant. In contrast, the band gap is significantly underestimated by PBE $(4.0 \mathrm{eV})$ while it is satisfactorily reproduced by B3LYP $(5.66 \mathrm{eV})$, in agreement with previous studies $[18,46]$. This result emphasizes the importance of using a hybrid functional scheme for the quantitative study of the electronic structure properties of diamond surfaces.

Previous theoretical studies of H-D surfaces have employed either GGA or LDA approximations [17, 40, 41] while B3LYP has only been used for unsaturated surfaces [19], due to its prohibitive computational cost using conventional planewave basis sets. However, the use of hybrid DFT functionals to study H-D surfaces and the effect of adsorbate molecules is missing and can provide a quantitative understanding of the electronic properties, especially with regard to the hole injection from adsorbates to diamond surfaces.

\subsection{Bare and hydrogenated diamond surfaces}

We now consider the structural and electronic properties of bare and hydrogenated (100), (110) and (111) diamond surfaces. The two dangling bonds per carbon atom in the (100) makes this surface very reactive. However, it has been shown experimentally that this surface undergoes a structural reconstruction that leads to the formation of C-C surface dimers [47, 48], thereby reducing the number of dangling bonds and lowering its energy. Thus, our calculations are performed on the $2 \times 1$ reconstructed surface cell. This system is typically labeled $\mathrm{D}(100)-2 \mathrm{x} 1$. The other diamond surfaces (labeled $\mathrm{D}(110)$ and $\mathrm{D}(111)$ ) present one dangling bond per carbon atom and therefore, do not need any reconstruction to stabilize.

Fig. 1 shows the relative displacements $(\Delta z)$ of the carbon layers along the $z$-axis after relaxation for the bare diamond surfaces. The first 7 layers are relaxed for the $\mathrm{D}(100)-2 \times 1$ and the first 5 layers for $\mathrm{D}(110)$ and $\mathrm{D}(111)$ surfaces. We found that the outer 4 layers are 

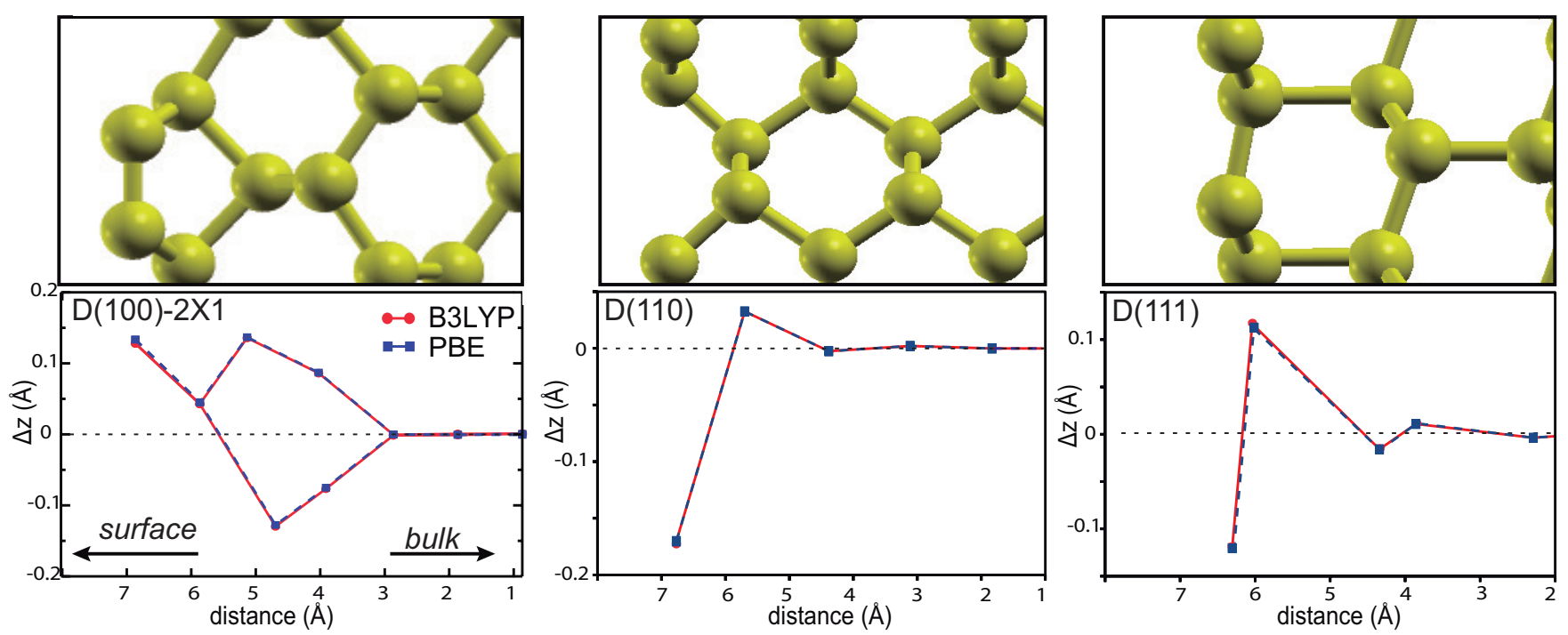

Figure 1. Atomic displacements along the $z$ direction of relaxed surface layers with respect to the bulk atomic crystallographic positions $\left(\Delta z=z_{\text {relaxed }}-z_{\text {bulk }}\right)$ for the reconstructed (100)-2x1, (110), and (111) bare diamond surfaces where the top and bottom 7, 5, and 5 surface layers are relaxed, respectively. In each case, the two innermost layers are fixed to bulk parameters to simulate the bulk conditions of diamond. The origin of the horizontal axis is in the center of the slab.

affected by the relaxation process for (100) and (111) surfaces while only the 2-3 first layers change for the (110) surface. Increasing the size of the slab does not change the energies of the surface states by more than a few meV. Similarly, neither the band gaps nor the atomic charges change appreciably, as can be seen also in Tables III, V, and VII.

Our simulations of the different bare diamond surfaces compare well with the structural properties reported by De La Pierre et al. in Ref. 19 who also employed the B3LYP functional. This agreement provides us the starting point for studying both the H-D surfaces and the influence of the adsorption molecules. It should be mentioned that there is a difference between our relaxation procedure and that employed in Ref. 19. While the authors of this previous work relaxed all the atomic positions and the cell parameters, we use bulk structural parameters and only relax the atomic positions of several surface layers, keeping the atoms in the central layers fixed to the bulk coordinates. Thus, small differences on the order of $0.5 \%$ have been found.

In the following discussion we present the structural and electronic results obtained for each bare and hydrogenated diamond surface.

\subsubsection{The reconstructed (100)-2x1 surface}

The reconstructed (100)-2x1 surface is one of the most important diamond surfaces since it can be easily grown using CVD [49, 50]. Table II contains the properties for both the $\mathrm{D}(100)-2 \times 1$ and the hydrogenated $\mathrm{D}(100)-2 \times 1$
Table II. C-C and C-H atomic distances $(\AA)$ and bond angles $\left(^{\circ}\right)$ of the bare and $\mathrm{H}-\mathrm{D}(100)-2 \times 1$ diamond surfaces when relaxing the outer 5 layers obtained via $\mathrm{PBE}$ and B3LYP calculations. See Fig. 2a for definition of parameters.

\begin{tabular}{ccccc} 
& \multicolumn{2}{c}{$\mathrm{D}(100)-2 \times 1$} & \multicolumn{2}{c}{ H-D $(100)-2 \times 1$} \\
\cline { 2 - 5 } & PBE & B3LYP & PBE & B3LYP \\
\hline$d_{01}$ & - & - & 1.102 & 1.093 \\
$d_{11}$ & 1.384 & 1.376 & 1.629 & 1.633 \\
$d_{12}$ & 1.515 & 1.518 & 1.547 & 1.546 \\
$d_{23 a}$ & 1.583 & 1.565 & 1.540 & 1.534 \\
$d_{23 b}$ & 1.578 & 1.601 & 1.580 & 1.587 \\
$d_{34 a}$ & 1.517 & 1.502 & 1.526 & 1.518 \\
$d_{34 b}$ & 1.583 & 1.568 & 1.572 & 1.569 \\
$\Delta z_{01}$ & - & - & 1.014 & 1.007 \\
$\Delta z_{12}$ & 0.683 & 0.686 & 0.814 & 0.815 \\
$\Delta z_{23}$ & 0.806 & 0.852 & 0.814 & 0.830 \\
$\Delta z_{23 *}$ & 0.257 & 0.184 & 0.181 & 0.155 \\
$\alpha_{0}$ & - & - & 113.08 & 112.94 \\
$\alpha_{1}$ & 108.49 & 108.62 & 103.49 & 103.42 \\
$\alpha_{1 *}$ & 113.12 & 112.77 & 109.69 & 109.72 \\
$\alpha_{2}$ & 93.90 & 93.47 & 99.33 & 99.24
\end{tabular}

(H-D(100)-2x1) surfaces using PBE and B3LYP density functionals. For this surface PBE and B3LYP geometry relaxations do not differ appreciably. When hydrogen atoms are introduced on the diamond surface (see Fig. $2 \mathrm{a})$, the crystal geometry changes significantly. Calculations using both DFT functionals show that hydrogens pull the diamond surface upward, thus increasing the distance between the first two surface carbon layers $\left(\Delta z_{12}\right)$. Furthermore, the C-C bond distance at the surface $\left(d_{1}\right)$ increases significantly upon hydrogenation from 1.376 to 

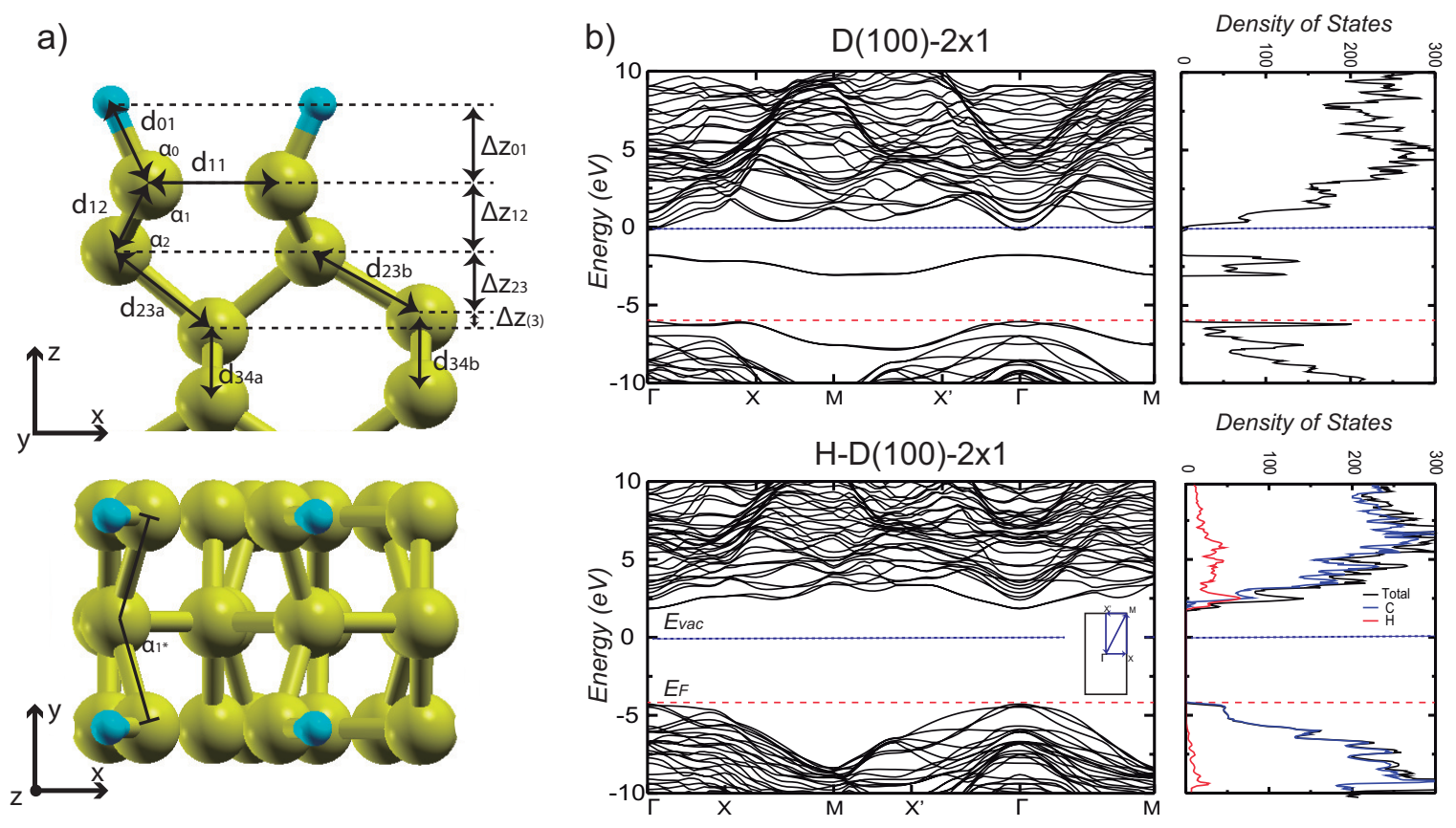

Figure 2. a) Side and top views of the H-D(100)-2x1 reconstructed surface. The distances and angles are reported in Table II. b) Electronic structure bands and projected density of states of the $\mathrm{D}(100)-2 \mathrm{x} 1$ and $\mathrm{H}-\mathrm{D}(100)-2 \mathrm{x} 1$ reconstructed surfaces obtained via B3LYP. Fermi energy and vacuum levels are displayed by dashed lines. The saturation of the surface by hydrogen removes surface states from the band gap and results in the vacuum level to be positioned inside the band gap, thereby demonstrating the onset of negative electron affinity.

$1.633 \AA$ (B3LYP case) similar to our PBE results and in agreement with Ref. 40. Note that bulk diamond C-C bond length is $1.546 \AA$. In addition, the $\alpha_{2}, \alpha_{1}$, and $\alpha_{1}{ }^{*}$ bond angles change as a consequence of the hydrogenation, increasing $\alpha_{2}$ by $\simeq 6 \%$ and reducing $\alpha_{1}$ and $\alpha_{1}{ }^{*}$ slightly. As expected, based on the diamond bulk structure results, PBE and B3LYP structural parameters do not differ significantly from each other.

We now consider the electronic properties of both the $\mathrm{D}(100)-2 \mathrm{x} 1$ and H-D(100)-2x1 surfaces. In Table III we present the results for properties such as surface energy, atomic net charges, surface band gap, $\mathrm{H}$ desorption energy, and electronic affinity obtained via PBE and B3LYP. To ensure a good convergence and accuracy of the calculated electronic properties of $\mathrm{H}-\mathrm{D}$ we also consider thicker slabs with up to 7 relaxed surface layers with the innermost 2 layers fixed to the bulk parameters. As can be seen in Table III, the electronic properties are well converged with 5 relaxed layers.

The double bonded $\mathrm{C}$ dimers at the surface of bare diamond introduce occupied $\pi$ and unoccupied $\pi{ }^{*}$ surface states into the fundamental band gap below and above the Fermi energy respectively (Fig. 2b). Covering the diamond surface with hydrogen atoms removes the $\pi$ bonded surface states from the bandgap and moves the $\pi^{*}$ surface states into the conduction band. As a consequence the additional electrons fill these gap states and the band gap increases by $3.1 \mathrm{eV}$ (from 2.9 to $6.0 \mathrm{eV}$ ) using B3LYP and $3.3 \mathrm{eV}$ (from 1.2 to $4.5 \mathrm{eV}$ ) by PBE (see Table III).

By means of Eq. 1 we calculated the surface energy per atom on bare diamond, where small differences of a few $\mathrm{meV}$ are observed when relaxing 5 or more layers. In the hydrogenation process, carbon net charges change from 5.837 to $6.047\left(q_{1}\right)$ and from 6.072 to $5.996\left(q_{2}\right)$ while the charge in hydrogen atoms becomes 0.886 (B3LYP). The first carbon layer contains excess of electrons while the hydrogens act as hole-doped sites. In this situation there is considerable band bending at the H-D surface that moves the vacuum level into the band gap (see Fig. $2 \mathrm{~b}$ ) and thus, leads to a NEA. The experimental NEA value is $-1.3 \mathrm{eV}$ [51], which is reasonably well described by B3LYP $(-1.01 \mathrm{eV})$ but not by PBE $(-0.05 \mathrm{eV})$. This highlights the importance of using hybrid functionals to study the electronic properties of diamond surfaces.

We use Eq. 2 to calculate the hydrogen desorption energy. B3LYP and PBE functionals provide a converged values of $\simeq 1.97 \mathrm{eV}$ and $\simeq 1.96 \mathrm{eV}$ respectively that compares well with the estimated experimental value of $4.16 \mathrm{eV}[44]$. From the above results we note that PBE and B3LYP produce similar bare and hydrogenated diamond surface structures as well as electronic band gap changes upon full hydrogen coverage. However, unlike PBE, B3LYP provides values that are in very good agreement with experiment concerning key properties associated with transport such as the band gap and NEA. 
Table III. Electronic properties of bare and hydrogenated (100)-2x1 diamond surface (units in eV, and $e$ ) when relaxing 5, 6, and 7 layers obtained via PBE and B3LYP calculations. $q_{0}$ indicates the $\mathrm{H}$ net charge while $q_{1}$ and $q_{2}$ indicates the $\mathrm{C}$ net charges of the first 2 layers of diamond. NEA* are the calculated NEA values using 'ghost' functions at the surface (following the method in Ref. 38).

\begin{tabular}{llccccccccccc} 
& \multicolumn{1}{c}{$\mathrm{D}(100)$} & \multicolumn{10}{c}{$\mathrm{H}-\mathrm{D}(100)$} \\
\cline { 2 - 13 } & \multicolumn{1}{c}{ Surf. E/atom } & $q_{1}$ & $q_{2}$ & Gap H desorp./at & $q_{0}$ & $q_{1}$ & $q_{2}$ & NEA NEA* Surf. Gap \\
\hline \multirow{2}{*}{5 layers } & PBE & 1.986 & 5.812 & 6.079 & 1.2 & 1.96 & 0.886 & 6.048 & 5.997 & -0.40 & -0.05 & 4.5 \\
& B3LYP & 1.986 & 5.837 & 6.072 & 2.9 & 1.97 & 0.897 & 6.047 & 5.996 & -1.46 & -1.01 & 6.0 \\
\hline \multirow{2}{*}{6 layers } & PBE & 1.979 & 5.812 & 6.082 & 1.2 & 1.95 & 0.886 & 6.047 & 5.999 & -0.40 & -0.05 & 4.5 \\
& B3LYP & 1.988 & 5.836 & 6.074 & 2.9 & 1.97 & 0.897 & 6.044 & 6.002 & -1.46 & -1.01 & 6.0 \\
\hline \multirow{2}{*}{7 layers } & PBE & 1.978 & 5.811 & 6.082 & 1.2 & 1.95 & 0.886 & 6.048 & 5.998 & -0.40 & -0.05 & 4.4 \\
& B3LYP & 1.988 & 5.836 & 6.074 & 2.9 & 1.97 & 0.897 & 6.044 & 6.002 & -1.46 & -1.01 & 6.0 \\
\hline
\end{tabular}

Table IV. C-C and C-H atomic distances (in $\AA$ ) and bond angles $\left({ }^{\circ}\right.$ ) of bare and $\mathrm{H}$ covered (110) diamond surface when relaxing the outer 3 layers obtained via PBE and B3LYP calculations. See also Fig. 3a.

\begin{tabular}{ccccc}
\multirow{2}{*}{$\mathrm{D}(110)$} & \multicolumn{2}{c}{$\mathrm{D}(110)$} & \multicolumn{2}{c}{$\mathrm{H}-\mathrm{D}(110)$} \\
\cline { 2 - 5 } & PBE & B3LYP & PBE & B3LYP \\
\hline$d_{01}$ & - & - & 1.103 & 1.092 \\
$d_{11}$ & 1.437 & 1.434 & 1.527 & 1.526 \\
$d_{12}$ & 1.481 & 1.480 & 1.535 & 1.534 \\
$d_{22}$ & 1.511 & 1.510 & 1.541 & 1.554 \\
$\Delta z_{01}$ & - & - & 0.928 & 0.920 \\
$\Delta z_{12}$ & 1.061 & 1.057 & 1.229 & 1.227 \\
$\Delta z_{23}$ & 1.300 & 1.301 & 1.299 & 1.300 \\
$\alpha_{0}$ & - & - & 110.45 & 110.61 \\
$\alpha_{1}$ & 123.24 & 123.64 & 111.80 & 111.85 \\
$\alpha_{2}$ & 113.60 & 113.63 & 110.32 & 110.33 \\
$\alpha_{3}$ & 100.18 & 100.05 & 107.57 & 107.57 \\
\hline
\end{tabular}

\subsubsection{The $\mathrm{D}(110)$ surface}

As commented above, the bare (110) diamond surface contains one dangling bond per carbon atom and thus, it does not have to reconstruct in order to form a stable surface. Fig. 3a displays the hydrogenated structure where the carbon atoms are arranged in zigzag chains. The crystal structural parameters are shown in Table IV for bare and hydrogenated surfaces.

When the surface is covered by a layer of hydrogen atoms, the inter-layer distance $\Delta z_{12}$ increases significantly by $\simeq 0.2 \AA$ (see Table IV). The H-bond formation is also responsible for the increase in bond distances and the reduction of bond angles, in agreement with Ref. [41]. This indicates that the carbon atoms at the surface have suffered a compressive strain along the $y$-direction (see Fig. 3a). We note that there is no significant difference between PBE and BL3LYP structural results, similar to the (100) case. Both functionals show that the surface energy per atom is smaller in $\mathrm{D}(110)$ than in the $\mathrm{D}(100)$ $2 \mathrm{x} 1$ by $\simeq 0.4 \mathrm{eV} / \mathrm{at}$, which indicates that this surface requires less energy to be formed.

Table V shows the electronic properties of $\mathrm{D}(110)$ and $\mathrm{H}-\mathrm{D}(110)$ surfaces, where we also considered the 4 and 5 relaxed layer cases to ensure convergence. The lone dan- gling bond per carbon atom at the $\mathrm{D}(110)$ surface forms $\pi$ antibonding states localized in the bandgap, thereby producing a metal. The surface carbon net charges change with the inclusion of hydrogen atoms similar to the $\mathrm{H}$ $\mathrm{D}(100)-2 \mathrm{x} 1$ case, where $q_{1}$ presents an excess of electrons and hydrogen atoms are hole-doped. The H-D(100) surface is found to be a semiconductor with a band gap of $5.3 \mathrm{eV}(3.7 \mathrm{eV})$, as obtained via B3LYP (PBE) when the diamond (110) surface is covered by a layer of hydrogen atoms (see Fig. 3b). The observed NEA in the hydrogenated structure is found to be $-1.85 \mathrm{eV}$ for B3LYP and $-0.95 \mathrm{eV}$ for PBE.

\subsubsection{The $\mathrm{D}(111)$ surface}

The (111) diamond surface is another possible candidate for forming a low energy surface structure under CVD conditions. This surface can have two different terminations, one exhibits one dangling bond while the other one has three dangling bonds per surface carbon atom. A number of possible reconstructions have been proposed [53]. Among these, the Pandey-chain reconstruction is currently the most accepted model [54]. Here, we study the ideal truncated bulk surface without reconstruction since it is the most energetically stable surface upon hydrogenation [55]. The $\mathrm{D}(111)$ structure has one dangling bond per carbon atom at the surface and corresponds to the natural cleavage plane of diamond. Fig. 4a displays the top and side views of the H-D(111) surface while Table VI contains the structural parameters before and after hydrogenation. Upon surface hydrogenation, $\Delta z_{12}$ increases significantly by more than $0.2 \AA$ while $\Delta z_{23}$ is reduced by almost $0.2 \AA$. As a consequence, the $d_{1}, d_{2}$, and $d_{3}$ bond distances become almost identical and close to the bulk diamond $\mathrm{C}$ bond length.

The electronic states of this structure are delocalized over the entire surface producing a conducting surface as found in the (110) case. The results presented in Table VII show the convergence of the electronic properties (surface energy, hydrogen desorption, electronic affinity and band gap). The role of hydrogen is to saturate the dangling bonds and as a consequence, the system be- 

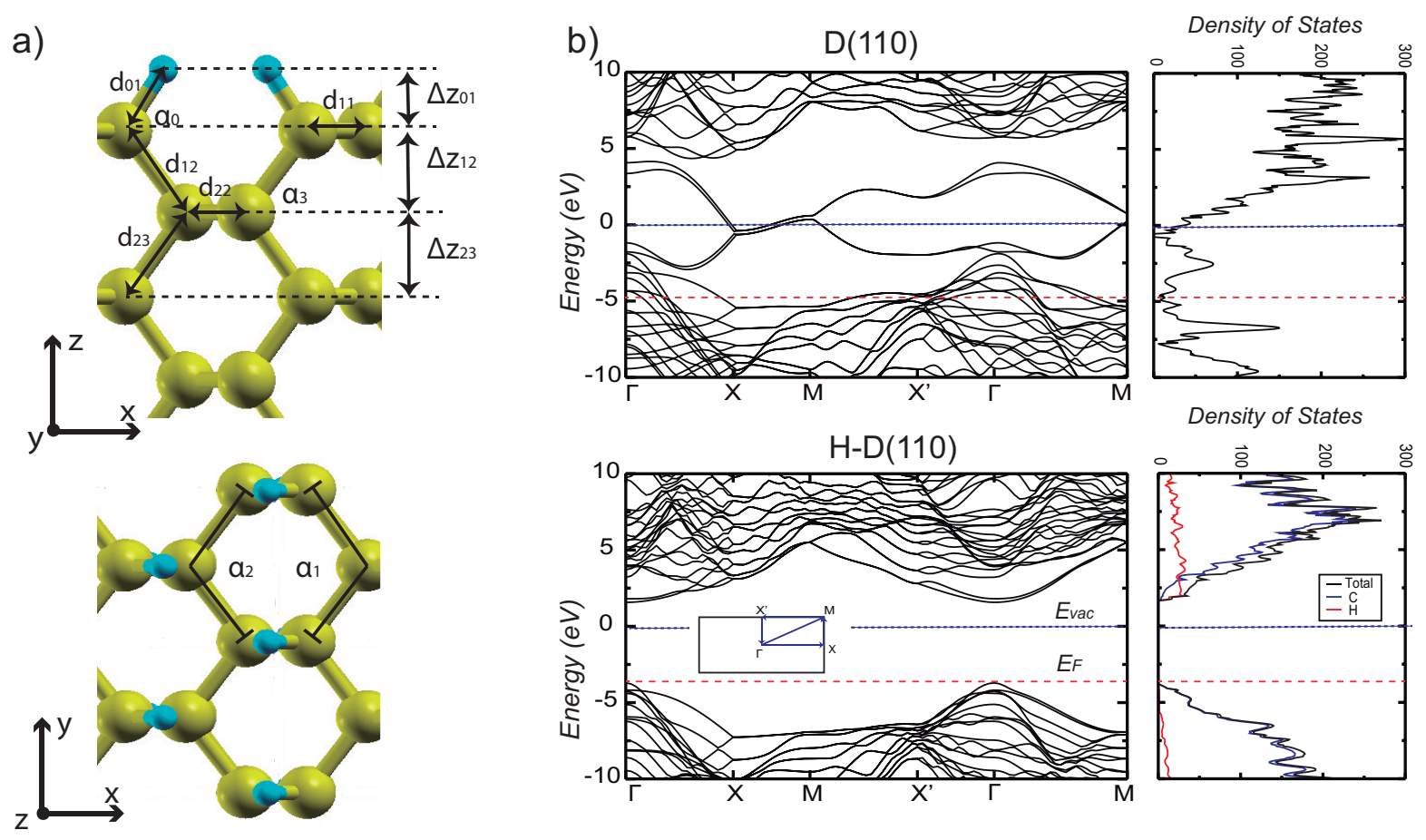

Figure 3. a) Side and top views of the H-D(110) surface. The distances and angles are reported in Table II. b) Electronic structure bands and projected density of states of the $\mathrm{D}(110)$ and $\mathrm{H}-\mathrm{D}(110)$ surfaces obtained via B3LYP. Fermi energy and vacuum levels are displayed by dashed lines. The saturation of the surface by hydrogen opens a significant band gap where the vacuum level is located, thereby showing negative electron affinity.

Table V. Electronic properties of bare and hydrogenated (110) diamond surface (units in eV, and $e$ ) when relaxing 3,4 , and 5 layers obtained via PBE and B3LYP calculations. NEA* are the calculated NEA values using 'ghost' functions at the surface (following the method in Ref. 38).

\begin{tabular}{|c|c|c|c|c|c|c|c|c|c|c|c|c|}
\hline & \multicolumn{4}{|c|}{$\mathrm{D}(110)$} & \multicolumn{7}{|c|}{$\mathrm{H}-\mathrm{D}(110)$} & \multirow[b]{2}{*}{ Surf. Gap } \\
\hline & & Surf. E/at & $q_{1}$ & $q_{2}$ & Gap & H desorp./at & $q_{0}$ & $q_{1}$ & $q_{2}$ & NEA & $\mathrm{NEA}^{*}$ & \\
\hline \multirow[t]{2}{*}{3 layers } & $\mathrm{PBE}$ & 1.533 & 5.879 & 6.121 & 0.0 & 1.75 & 0.879 & 6.081 & 6.037 & -1.0 & -0.95 & 3.7 \\
\hline & B3LYP & 1.604 & 5.894 & 6.103 & 0.0 & 1.85 & 0.892 & 6.070 & 6.032 & -1.9 & -1.85 & 5.3 \\
\hline \multirow[t]{2}{*}{4 layers } & PBE & 1.536 & 5.879 & 6.120 & 0.0 & 1.76 & 0.879 & 6.081 & 6.037 & -1.0 & -0.95 & 3.7 \\
\hline & B3LYP & 1.608 & 5.894 & 6.101 & 0.0 & 1.87 & 0.892 & 6.068 & 6.035 & -1.9 & -1.85 & 5.3 \\
\hline \multirow[t]{2}{*}{5 layers } & $\mathrm{PBE}$ & 1.536 & 5.879 & 6.119 & 0.0 & 1.76 & 0.879 & 6.081 & 6.037 & -1.0 & -0.95 & 3.7 \\
\hline & B3LYP & 1.608 & 5.894 & 6.101 & 0.0 & 1.87 & 0.892 & 6.067 & 6.035 & -1.9 & -1.85 & 5.2 \\
\hline
\end{tabular}

comes semiconducting (Fig. 4b). For full surface hydrogen coverage, the hydrogen atoms lose 0.1 electron and become slightly hole-doped. Experimentally the NEA value is $-1.27 \mathrm{eV}$ [56] with B3LYP producing a value of $-1.42 \mathrm{eV}$ that is closer to the experimental value than the provided by $\mathrm{PBE}$, which is $-0.7 \mathrm{eV}$.

Summarizing this section, we have observed that while calculated structural parameters with either PBE or B3LYP are in good agreement, the electronic properties differ significantly. B3LYP provides larger band gaps and larger NEA than PBE, which compare well with experimental measurements. Therefore, we choose the B3LYP approximation for studying the surface properties of H-D in the presence of adsorbates. We recall the reader that the use of the crystal slab model permits to accurately calculate the difference in energetics between the vacuum level and the low lying excited states. This allows us to systematically study the charge transfer effect produced by the adsorption of molecular species.

\subsection{Study of adsorbates on H-D surfaces}

To have hole conductivity on H-D surfaces, holes need to be injected via functionalization using specific adsorbate molecules. The effect of different molecular species 

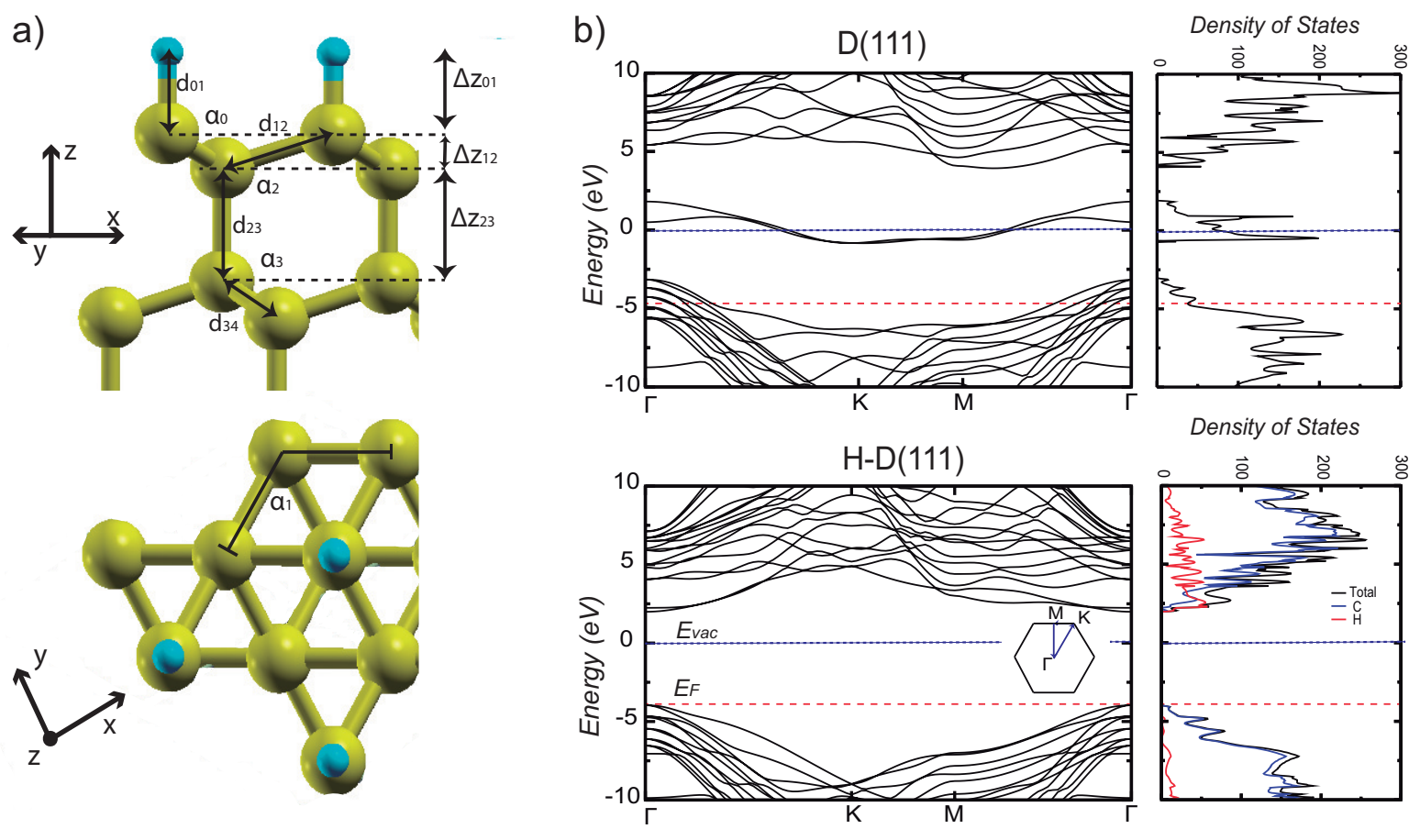

Figure 4. a) Side and top views of the H-D(111) reconstructed surface. The distances and angles are reported in Table II. b) Electronic structure bands and projected density of states of the $\mathrm{D}(111)$ and H-D(111) surfaces obtained via B3LYP. Fermi energy and vacuum levels are displayed by dashed lines. Similar to the H-D(100) surface, the saturation by surface hydrogen removes surface states from the inside of the band gap and leads to the appearance of negative electron affinity.

Table VI. C-C and C-H atomic distances (in $\AA$ ) and bond angles $\left(^{\circ}\right)$ of the bare and $\mathrm{H}$ covered (111) diamond surface obtained via PBE and B3LYP calculations. See also Fig. 4a.

\begin{tabular}{ccccc} 
& \multicolumn{2}{c}{$\mathrm{D}(111)$} & \multicolumn{2}{c}{$\mathrm{H}-\mathrm{D}(111)$} \\
\cline { 2 - 5 } & PBE & B3LYP & PBE & B3LYP \\
\hline$d_{01}$ & - & - & 1.088 & 1.082 \\
$d_{12}$ & 1.484 & 1.484 & 1.535 & 1.536 \\
$d_{23}$ & 1.691 & 1.696 & 1.529 & 1.532 \\
$d_{34}$ & 1.538 & 1.538 & 1.547 & 1.547 \\
$\Delta \mathrm{z}_{01}$ & - & - & 1.088 & 1.082 \\
$\Delta \mathrm{z}_{12}$ & 0.270 & 0.267 & 0.476 & 0.477 \\
$\Delta \mathrm{z}_{23}$ & 1.691 & 1.696 & 1.529 & 1.532 \\
$\Delta \mathrm{z}_{34}$ & 0.487 & 0.486 & 0.515 & 0.514 \\
$\alpha_{0}$ & - & - & 108.2 & 108.0 \\
$\alpha_{1}$ & 116.75 & 116.83 & 110.8 & 110.7 \\
$\alpha_{2 a}$ & 108.49 & 108.47 & 108.0 & 108.2 \\
$\alpha_{2 a}$ & 110.44 & 110.45 & 109.4 & 109.5 \\
\hline
\end{tabular}

adsorbed on $\mathrm{H}-\mathrm{D}(100)$, $\mathrm{H}-\mathrm{D}(110)$, and $\mathrm{H}-\mathrm{D}(111)$ is investigated in this section. Specifically we consider NO, $\mathrm{NO}_{2}$, and $\mathrm{O}_{3}$, since they have all been proposed to be potential (electron) acceptor molecules which could lead to hole accumulation on H-D surfaces [17]. Fig. 5 shows the relaxed structures upon adsorption of the different molecules, as calculated using the B3LYP functional. Depending on the H-D surface, each molecule is arranged at a specific adsorption site in order to reach the lowest formation energy, that is, avoiding being right on top of
$\mathrm{H}$ atoms to reduce repulsion. In addition to the electron transfer from the hydrogen layer to the diamond surface discussed in the previous section, there is an additional charge transfer of electrons from the hydrogen surface layer to the adsorbate molecule. These charge transfers are generated by the electric field present between the diamond slab, hydrogen layer, and the adsorbate molecule. The resulting electrostatic potential confines the holes to a small region centered on the hydrogen layer where it forms a quasi two-dimensional hole gas.

Table VIII summarizes the atomic charges, charge transfers $(\Delta q)$, cohesive energies, calculated hole densities $(p s)$, and band bendings for each combination of adsorbate molecule and H-D surface considered. We note that the adsorption of $\mathrm{NO}_{2}$ and $\mathrm{O}_{3}$ molecules on $\mathrm{H}-\mathrm{D}$ surfaces has a significant impact on $\Delta q$. This leads to the appearance of band bending at the interface due to the abrupt variation of the electrostatic potential. It follows, as can be seen in Fig. 6, that the diamond contribution to the density of states crosses the Fermi level and the system becomes metallic. For $\mathrm{NO}$ adsorption, the $\Delta q$ on hydrogen atoms is very small, indicating very little hole creation. In this case, the diamond contribution to the density of states does not cross the Fermi level and therefore, the H-D surface remains semiconducting. The amount of upward band bending can be obtained from the calculated DOS (Fig. 6), and is provided in Table VIII. 
Table VII. Electronic properties of bare and hydrogenated $\mathrm{D}(111)$ surface (units in eV, and $e$ ) when relaxing 4 , 5, and 6 layers obtained via PBE and B3LYP calculations. NEA* are the calculated NEA values using 'ghost' functions at the surface (following the method in Ref. 38).

\begin{tabular}{cccccccccccc} 
& \multicolumn{1}{c}{$\mathrm{D}(111)$} & \multicolumn{10}{c}{$\mathrm{H}-\mathrm{D}(111)$} \\
\cline { 2 - 12 } & Surf. E/at & $q_{1}$ & $q_{2}$ & Gap & H desorp./at & $q_{0}$ & $q_{1}$ & $q_{2}$ & NEA NEA* Surf. Gap \\
\hline 4 layers PBE & 2.031 & 5.703 & 6.278 & 0.0 & 2.32 & 0.895 & 5.951 & 6.141 & -0.70 & -0.70 & 4.5 \\
B3LYP & 2.275 & 5.746 & 6.238 & 0.0 & 2.62 & 0.906 & 5.953 & 6.127 & -1.66 & -1.42 & 6.0 \\
\hline 5 layers PBE & 2.030 & 5.702 & 6.279 & 0.0 & 2.34 & 0.898 & 5.960 & 6.133 & -0.7 & -0.70 & 4.5 \\
B3LYP & 2.273 & 5.745 & 6.239 & 0.0 & 2.63 & 0.909 & 5.957 & 6.124 & -1.66 & -1.42 & 6.0 \\
\hline 6 layers PBE & 2.031 & 5.702 & 6.279 & 0.0 & 2.34 & 0.898 & 5.961 & 6.133 & -0.7 & -0.70 & 4.5 \\
B3LYP & 2.273 & 5.746 & 6.239 & 0.0 & 2.63 & 0.909 & 5.958 & 6.124 & -1.66 & -1.42 & 6.0 \\
\hline
\end{tabular}

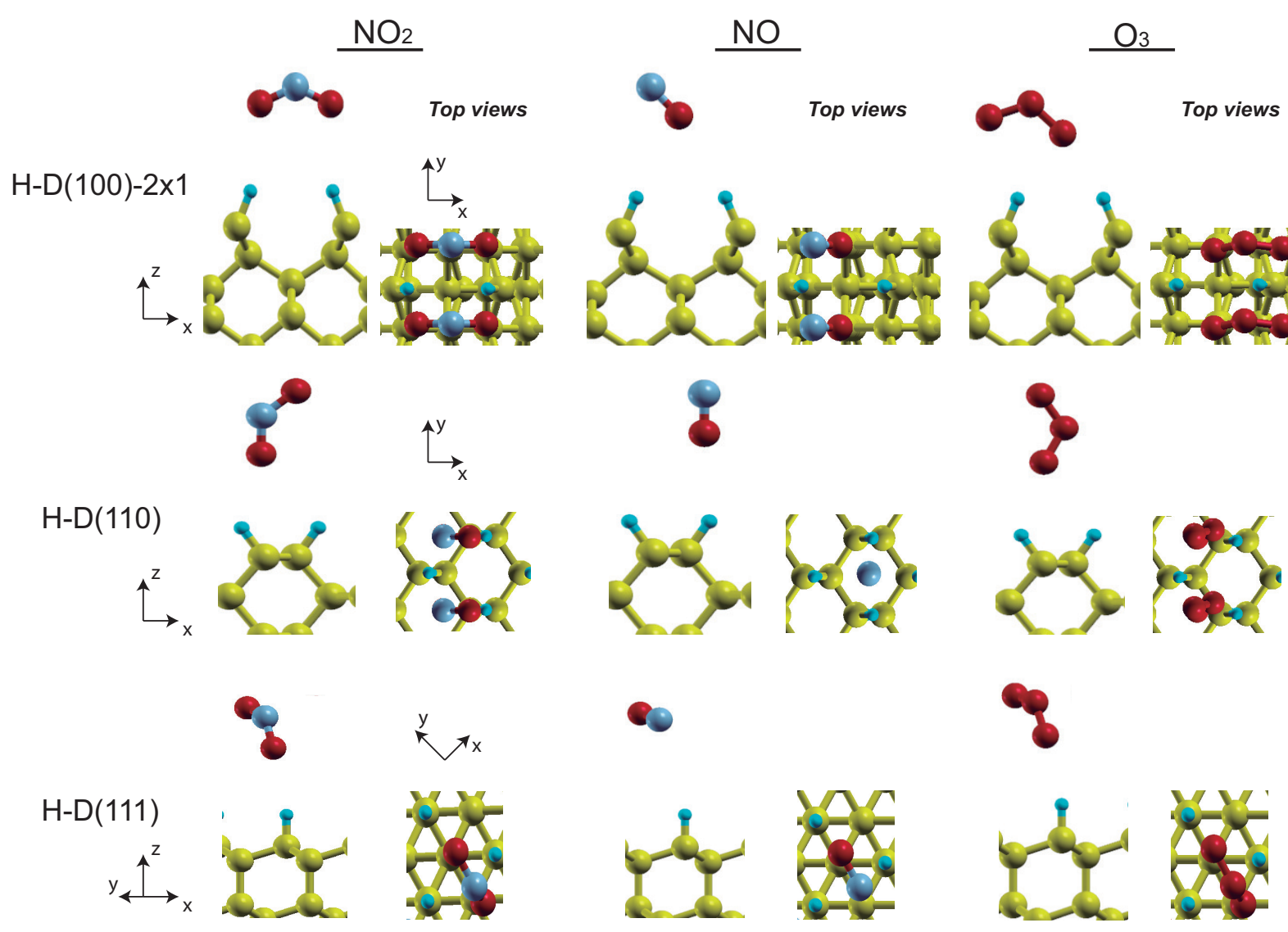

Figure 5. Relaxed structures of $\mathrm{NO}_{2}$, NO, and $\mathrm{O}_{3}$ adsorbates on the $\mathrm{H}-\mathrm{D}$ (100), (110), and (111) surfaces obtained via hybrid functional B3LYP (side and top views). Hydrogen, carbon, nitrogen, and oxygen atoms are shown in dark blue, yellow, light blue, and red color, respectively.

Our calculations using B3LYP hybrid functional have the accuracy to quantify the $\Delta q$ and the hole density $(p s)$ for each adsorbed molecule on H-D surfaces. We note that $p s$ depends on both the local symmetry of the surface and the type of adsorbed molecule. Regardless of the adsorbate considered, the surfaces that produce higher $p s$ are arranged as: $p s_{\mathrm{H}-\mathrm{D}(110)}>p s_{\mathrm{H}-\mathrm{D}(111)}>p s_{\mathrm{H}-\mathrm{D}(100)}$, which is in agreement with the experimental work reported in Ref. 57. The largest ps occurs for $\mathrm{NO}_{2}$ on H-D(110) among the systems studied. As pointed out in Ref. 7 and Ref. 17 the charge transfer between adsorbate and H-D is triggered when the lowest unoccu- pied molecular orbital (LUMO) approaches the VBM. A qualitative result can be directly extracted from Fig. 7, where the H-D VBM and CBM levels and the HOMO and LUMO levels for different adsorbate molecules are plotted. When the LUMO level of the adsorbate is above the VBM of the H-D surface no charge transfer occurs (e.g., $\mathrm{NO}_{3}$ and $\mathrm{H}_{2} \mathrm{O}$ ), therefore no hole concentration is produced. As an approximation, the larger the difference between the H-D VBM and the adsorbate LUMO, the larger the charge transfer is, which leads to a larger band bending at the interface. It can be noted that H-D110 is the surface with higher VBM and therefore, the one that 


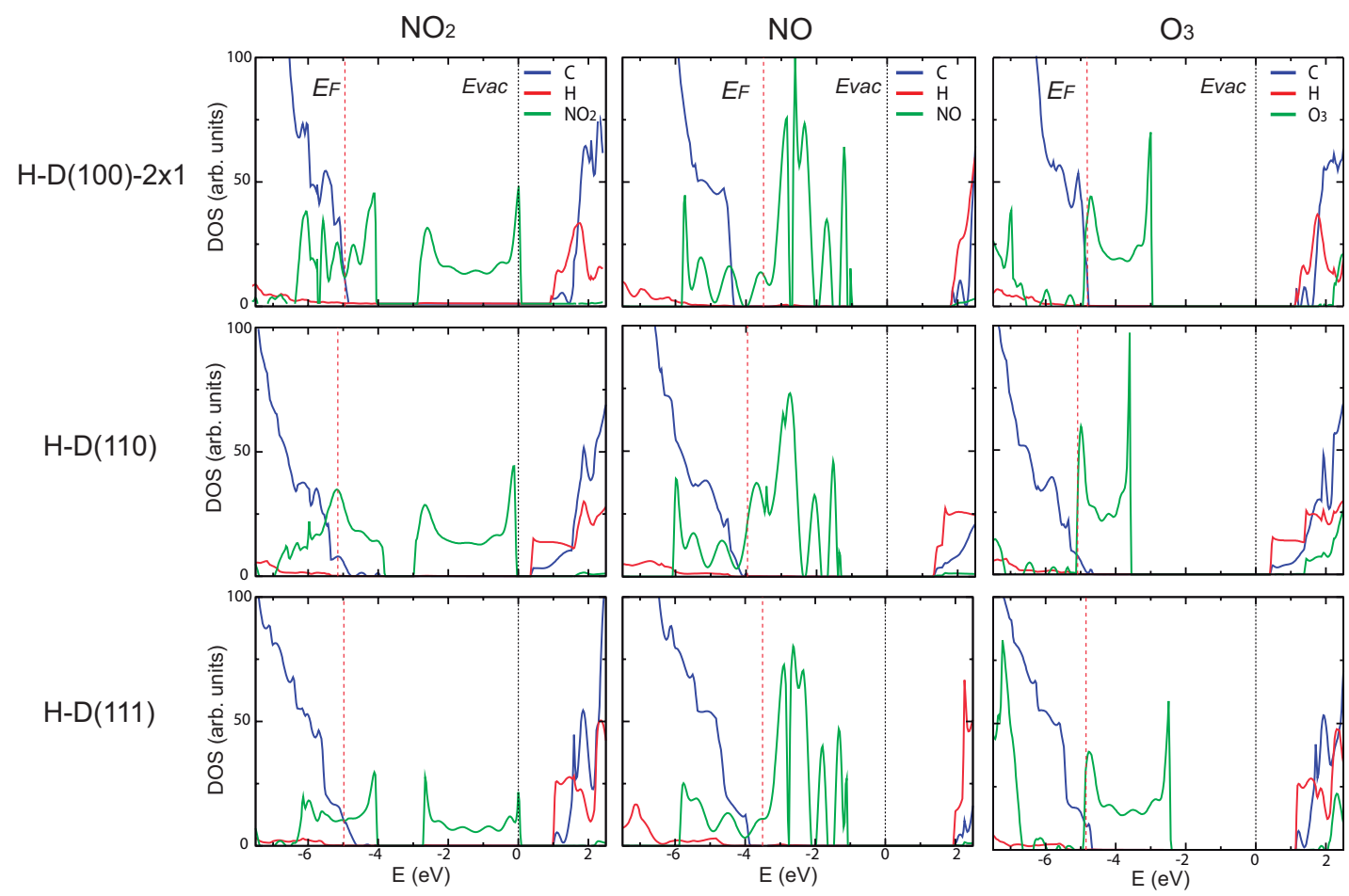

Figure 6. Projected density of states of $\mathrm{C}, \mathrm{H}$, and $\mathrm{NO}_{2}, \mathrm{NO}$, and $\mathrm{O}_{3}$ adsorbate molecules of the relaxed structures displayed in Fig. 5 obtained via B3LYP. In each case, the Fermi energy and vacuum levels are shown by red and blue lines, respectively.

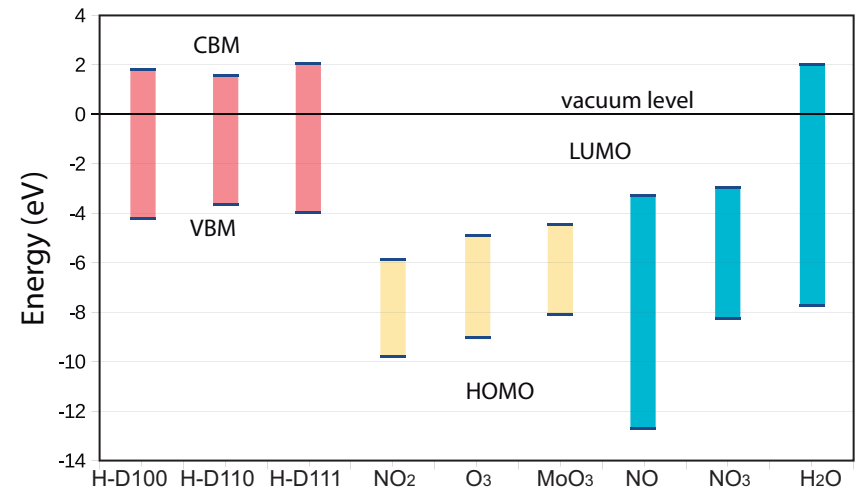

Figure 7. Schematic illustration of the valence band maximum (VBM) and conduction band minimum (CBM) of H-D surfaces and the highest molecular orbital (HOMO) and the lowest molecular orbital (LUMO) levels of different adsorbate molecules calculated via B3LYP. Vacuum level is set to $0 \mathrm{eV}$. To efficiently inject holes into the hydrogenated diamond surface, the LUMO state of the adsorbate must lie below (or close) the surface's VBM, as it is the case for $\mathrm{NO}_{2}, \mathrm{O}_{3}$, and $\mathrm{MoO}_{3}$. In contrast, $\mathrm{NO}, \mathrm{NO}_{3}$, and $\mathrm{H}_{2} \mathrm{O}$ are not good candidates for hole doping, for any of the surfaces considered.

produces more charge transfer among the H-D surfaces considered. However, to be fully predictive such a model should consider other parameters such as the $\mathrm{H}$ density at the surface.

\section{CONCLUSION}

We have used the B3LYP hybrid functional and all electron Gaussian-type orbital basis sets to perform a quantitatively accurate study of the structural and electronic properties of bare and hydrogen terminated (100), (110), and (111) diamond surfaces. Besides the structural results, which are in good agreement with previous theoretical studies, we provide for the first time the electronic properties of H-D surfaces using B3LYP, in good agreement with experiment. In addition, we have studied the adsorption of acceptor molecules on H-D surfaces and found that the combination of surface termination and molecule species is key to controlling the hole doping on diamond, where the highest $p s$ is obtained when $\mathrm{NO}_{2}$ is adsorbed on H-D(110). More importantly, we provide a qualitative study to estimate the electronic charge transfer between adsorbates and H-D surfaces. These results may assist future experimental searches for hydrogenated diamond based devices.

The computational work conducted by P.R and W. A. S. is supported by the U.S. Department of Energy under EPSCoR Grant No. DE-SC0012432 with additional support from the Louisiana Board of Regents and by an allocation of computing time from the Louisiana State University High Performance Computing center. V. M. acknowledges support by New York State under NYSTAR program C080117. 
Table VIII. Calculated adsorbate and $\mathrm{H}$ charge transfers, charges of the first and second surface diamond layers $(e)$, hole density of carriers ps $\left(\mathrm{x} 10^{13} / \mathrm{cm}^{2}\right)$, cohesion energy, and band bending $(\mathrm{eV})$ when $\mathrm{NO}_{2}, \mathrm{NO}$, and $\mathrm{O}_{3}$ molecules are adsorbed by the (100), (110), and (111) H-D surfaces via B3LYP.

\begin{tabular}{lccccccccc} 
& \multicolumn{3}{c}{$\mathrm{H}-\mathrm{D}(100)$} & \multicolumn{3}{c}{$\mathrm{H}-\mathrm{D}(110)$} & \multicolumn{3}{c}{$\mathrm{H}-\mathrm{D}(111)$} \\
\cline { 2 - 10 } & $\mathrm{NO}_{2}$ & $\mathrm{NO}$ & $\mathrm{O}_{3}$ & $\mathrm{NO}_{2}$ & $\mathrm{NO}$ & $\mathrm{O}_{3}$ & $\mathrm{NO}_{2}$ & $\mathrm{NO}$ & $\mathrm{O}_{3}$ \\
\hline$\Delta q$ adsorbate & 0.025 & -0.014 & 0.048 & 0.051 & 0.004 & 0.046 & 0.045 & -0.002 & 0.044 \\
$\Delta q \mathrm{H}$ & -0.053 & -0.001 & -0.088 & -0.103 & -0.012 & -0.094 & -0.101 & -0.002 & -0.108 \\
$q_{1}$ & 6.054 & 6.052 & 6.064 & 6.096 & 6.078 & 6.095 & 5.985 & 5.958 & 5.982 \\
$q_{2}$ & 6.002 & 5.998 & 6.000 & 6.034 & 6.034 & 6.033 & 6.126 & 6.126 & 6.132 \\
$p s$ & 4.15 & 0.07 & 6.89 & 11.28 & 1.27 & 10.19 & 7.89 & 0.12 & 8.39 \\
Exp. ps [57] & $8.9-9.8$ & - & - & $15.4-17.2$ & - & - & $14.5-15.2$ & - & - \\
Cohesion E & 1.73 & 2.29 & -0.21 & 1.71 & 1.10 & -0.18 & 1.71 & 0.93 & -0.70 \\
Band bending & 0.2 & 0.0 & 0.1 & 0.4 & 0.0 & 0.45 & 0.35 & 0.0 & 0.2 \\
\hline
\end{tabular}

* Corresponding author. Tel: 832-205-2528 E-mail: jprivero@lsu.edu

[1] H. Umezawa, M. Nagase, Y. Kato, S. Shikata, High temperature application of diamond power device, Diamond Relat. Mater. 24 (2012) 201.

[2] M. Willander, M. Friesel, Q.-U. Wahab, B. Straumal, Silicon carbide and diamond for high temperature device applications, J. Mater. Sci.- Mater. Electron. 17 (2006) 1.

[3] J. Isberg, J. Hammersberg, E. Johansson, T. Wikström, D. J. Twitchen, A. J. Whitehead et al., High carrier mobility in single-crystal plasma-deposited diamond, Science 297 (2002) 1670.

[4] C. J. H. Wort, C. S. J. Pickles, A. C. Beale, C. G. Sweeney, M. R. McClymont, R. J. Saunders et al., Recent advances in the quality of CVD diamond optical components, Proc. SPIE 3705 (1999) 119.

[5] R. J. Nemanich, J. A. Carlisle, A. Hirata, K. Haenen, CVD diamond-Research, applications, and challenges, MRS Bulletin 39 (2014) 490.

[6] M. I. Landstrass, K. V. Ravi, Resistivity of chemical vapor deposited diamond films, Appl. Phys. Lett. 55 (1989) 975.

[7] F. Maier, M. Riedel, B. Mantel, J. Ristein, L. Ley, Origin of surface conductivity in diamond, Phys. Rev. Lett. 85 (2000) 3472.

[8] P. Strobel, M. Riedel, J. Ristein, L. Ley, Surface transfer doping of diamond, Nature 430 (2004) 439.

[9] V. Chakrapani, J. C. Angus, A. B. Anderson, S. D. Wolter, B. R. Stoner, G. U. Sumanasekera, Charge transfer equilibria between diamond and an aqueous oxygen electrochemical redox couple, Science 318 (2007) 1424.

[10] J. van der Weide, Z. Zhang, P. K. Baumann, M. G. Wensell, J. Bernholc, R. J. Nemanich, Negative-electronaffinity effects on the diamond (100) surface, Phys. Rev. B 50 (1994) 5803(R).

[11] F. Maier, J. Ristein, L. Ley, Electron affinity of plasmahydrogenated and chemically oxidized diamond (100) surfaces, Phys. Rev. B 64 (2001) 165411.

[12] A. Bellucci, P. Calvani, M. Girolami, D. M. Trucchi, Environment and Electrical Engineering (EEEIC), IEEE 15th International Conference on, (2015) 1616.

[13] F. Maier, M. Riedel, B. Mantel, J. Ristein, L. Ley, Origin of surface conductivity in diamond, Phys. Rev. Lett. 85 (2000) 3472 .
[14] P. Strobela, J. Ristein, L. Leya, K. Seppeltb, I. V. Goldtc, O. Boltalina, Surface conductivity induced by fullerenes on diamond: Passivation and thermal stability, diamond Rel. Mat. 15 (2006) 720.

[15] A. D. Becke, A new mixing of Hartree-Fock and local density-functional theories, J. Chem. Phys. 98 (1993) 1372 .

[16] A. D. Becke, Density-functional thermochemistry. III. The role of exact exchange, J. Chem. Phys 98 (1994) 5648.

[17] Y. Takagi, K. Shiraishi, M. Kasu, H. Sato, Mechanism of hole doping into hydrogen terminated diamond by the adsorption of inorganic molecule, Surf. Sci. 609 (2013) 203.

[18] Y. Matsuda, J. Tahir-Khali, W. A. Goddard, Definitive band gaps for single-wall carbon nanotubes, J. Phys. Chem. Lett. 1 (2010) 2946.

[19] M. De La Pierre, M. Bruno, C. Manfredotti, F. Nestola, M. Prencipe, C. Manfredotti, The (100), (111) and (110) surfaces of diamond: an ab initio B3LYP study, Mol. Phys. 112 (2014) 1030.

[20] M. Bruno, F. R. Massaro, M. Prencipe, R. Demichelis, M. De La Pierre, F. Nestola, Ab Initio calculations of the main crystal surfaces of forsterite $\left(\mathrm{Mg}_{2} \mathrm{SiO}_{4}\right)$ : A preliminary study to understand the nature of geochemical processes at the Olivine interface, J. Phys. Chem. C 118 (2014) 2498.

[21] A. Damin, R. Dovesi, A. Zecchina, P. Ugliengo, CO/MgO (001) at different CO coverages: a periodic ab initio Hartree-Fock and B3LYP study, Surf. Sci. 479 (2001) 255.

[22] A. Rimola, M. Sodupe, S. Tosoni, B. Civarelli, P. Ugliengo, Interaction of Glycine with Isolated hydroxyl groups at the silica surface: First principles B3LYP periodic simulation, Langmuir 22 (2006) 6593.

[23] R. Demichelis, M. Bruno, F. R. Massaro, M. Prencipe, M. De La Pierre, F. Nestola, First-principle modelling of forsterite surface properties: Accuracy of methods and basis sets, J. Comput. Chem 36 (2015) 1439.

[24] R. Dovesi, R. Orlando, A. Erba, C. M. Zicovich-Wilson, B. Civalleri, S. Casassa et al., CRYSTAL14: A program for the ab initio investigation of crystalline solids, Int. J. Quantum Chem. 114 (2014) 1287.

[25] R. Dovesi, V. R. Saunders, C. Roetti, R. Orlando, C. M. Zicovich-Wilson, F. Pascale et al., CRYSTAL14 User's Manual, University of Torino, Torino (2014).

[26] J. P. Perdew, K. Burke, M. Ernzerhof, Generalized gradient approximation made simple, Phys. Rev. Lett. 77 
(1996) 3865.

[27] S. H. Vosko, L. Wilk, M. Nusair, Accurate spindependent electron liquid correlation energies for local spin density calculations: a critical analysis, Can. J. Phys. 58 (1980) 1200.

[28] A. D. Becke, Density-functional exchange-energy approximation with correct asymptotic behavior, Phys. Rev. A 38 (1988) 3098.

[29] C. Lee, W. Yang, R. G. Parr, Development of the ColleSalvetti correlation-energy formula into a functional of the electron density, Phys. Rev. B 37 (1988) 785.

[30] L. Ge, B. Montanari, J. H. Jefferson, D. G. Pettifor, N. M. Harrison, G. A. D. Briggs, Modeling spin interactions in carbon peapods using a hybrid density functional theory, Phys. Rev. B 77 (2008) 235416.

[31] http://www.crystal.unito.it/basis - sets.php

[32] H. J. Monkhorst, J. D. Pack, Special points for Brillouinzone integrations, Phys. Rev. B 13 (1976) 5188.

[33] R. S. Mulliken, Electronic population analysis on LCAOMO molecular wave functions, J. Chem. Phys. 23 (1955) 1833

[34] H. B. Jansen, P. Ros, Non-empirical molecular orbital calculations on the protonation of carbon monoxide, Chem. Phys. Lett. 3 (1969) 140.

[35] B. Liu, A. D. McLean, Accurate calculation of the attractive interaction of two ground state helium atoms, J. Chem. Phys. 59 (1973) 4557.

[36] S. Simon, M. Duran, J. J. Dannenberg, How does basis set superposition error change the potential surfaces for hydrogen bonded dimers?, J. Chem. Phys., 105 (1996) 11024.

[37] S. F. Boys and F. Bernardi, Calculation of Small Molecular Interactions by Differences of Separate Total Energies - Some Procedures with Reduced Errors, Mol. Phys., 19 (1970) 553.

[38] K. Doll, Calculation of the work function with a local basis set, Surf. Sci. 600 (2006) L321.

[39] I. Pino, M. Causá, V. Barone, Bottom-Up approach to innovative memory devices: II. molecular adsorption on electrodes and the asymmetric response, J. Chem. Phys. C 114 (2010) 21439.

[40] J. Furthmüller, J. Hafner, G. Kresse, Dimer reconstruction and electronic surface states on clean and hydrogenated diamond (100) surfaces, Phys. Rev. B 53 (1996) 7334 .

[41] G. Kern, J. Hafner, Ab initio calculations of the atomic and electronic structure of clean and hydrogenated diamond (110) surfaces, Phys. Rev. B 56 (1997) 4203.

[42] K. M. Odonnell, T. L. Martin, N. A. Fox, D. Cherns, Ab initio investigation of lithium on the diamond C (100) surface, Phys. Rev. B 82 (2010) 115303.

[43] M. J. Rutler, J. Robertson, Ab initio calculation of electron affinities of diamond surfaces, Phys. Rev. B 57 (1998) 9241.

[44] S. J. Sque, R. Jover, P. R. Briddon, Hydrogenation and oxygenation of the (100) diamond surface and the consequences for transfer doping, Phys. Stat. Sol. 202 (2005) 2091.

[45] J. Ristein, Surface science of diamond: Familiar and amazing, Surf. Sci. 600 (2006) 3677.

[46] X. Liu, L. Li, Q. Li, Y. Li, F. Lu, Optical and mechanical properties of $\mathrm{C}, \mathrm{Si}, \mathrm{Ge}$, and $3 \mathrm{C}-\mathrm{SiC}$ determined by first-principles theory using Heyd-Scuseria-Ernzerhof functional, Materials Science in Semiconductor Processing 16 (2013) 1369.

[47] B. B. Pate, The diamond surface: atomic and electronic structure, Surf. Sci. 165 (1986) 83.

[48] A. V. Hamza, G. D. Kubiak, R. H. Stulen, Hydrogen chemisorption and the structure of the diamond $\mathrm{C}(100)$ (2x1) surface, Sur. Sci. 237 (1990) 35.

[49] Y. L. Yang, M. P. D'Evelyn, Theoretical studies of clean and hydrogenated diamond (100) by molecular mechanics, J. Vac. Sci. Technol. A 10 (1992) 978.

[50] C. J. Chu, M. P. d'Evelyn, R. H. Hauge, J. L. Margrave, Mechanism of diamond growth by chemical vapor deposition on diamond (100), (111), and (110) surfaces: Carbon-13 studies, J. Appl. Phys. 70 (1991) 1695.

[51] F. Maier, J. Ristein, L. Ley, Electron affinity of plasmahydrogenated and chemically oxidized diamond (100) surfaces, Phys. Rev. B 64 (2001) 165411.

[52] D. D. Koleske, S. M. Gates, B. D. Thoms, J. N. Russell Jr., J. E. Butler, Isothermal desorption of hydrogen from polycrystalline diamond films, Surf. Sci. 320 (1994) L105.

[53] A. Scholze, W. G. Schmidt, F. Bechstedt, Structure of the diamond (111) surface: Single-dangling-bond versus triple-dangling-bond face, Phys. Rev. B 53 (1996) 13725.

[54] K.C. Pandey, New $\pi$-Bonded chain model for $\mathrm{Si}(111)-(2$ $\times$ 1) surface, Phys. Rev. Lett. 47 (1981) 1913.

[55] D. R. Alfonso, D. A. Drabold, S. E. Ulloa, Structural, electronic, and vibrational properties of diamond (100), (111), and (110) surfaces from ab initio calculations, Phys. Rev. B 51 (2005) 14669.

[56] J. B. Cui, J. Ristein, L. Ley, Electron affinity of the bare and hydrogen covered single crystal diamond (111) surface, Phys. Rev. Lett. 81 (1998) 429.

[57] H. Sato, M. Kasu, Maximum hole concentration for hydrogen-terminated diamond surfaces with various surface orientations obtained by exposure to highly concentrated $\mathrm{NO}_{2}$, Diamond Relat. Mater. 31 (2013) 47. 